\title{
Dermal Hemorrhage: A Clue to Lichen Sclerosus et Atrophicus
}

\author{
Michael Phan ${ }^{1}$, Emily Sou ${ }^{1}$, Ghadah Al Sannaa ${ }^{1}$, Melissa Erwin ${ }^{2}$, Ramon Sanchez ${ }^{1}$ \\ 1. Dermatology, University of Texas Medical Branch, Galveston, USA 2. Dermatology, Medical and Cosmetic \\ Dermatology, El Campo, USA
}

Corresponding author: Michael Phan, michaelbnphan@gmail.com

\begin{abstract}
Lichen sclerosus et atrophicus (LSA) may present in a rare bullous and hemorrhagic form that is often difficult to recognize both clinically and histopathologically. Clinically, the lesions may be characterized by atrophic and ivory-white sclerotic plaques in both genital and extragenital regions. Histologically, fully developed lesions of LSA are characterized by a thinned, effaced epidermis with interface change, a wide band of hyalinization in the upper dermis, and a lymphohistiocytic infiltrate below the hyalinized area. Extensive vacuolar degeneration weakens the integrity of the dermoepidermal junction, which contributes to the development of marked edema in the papillary dermis and subepidermal vesiculation. With increased fragility of dermal capillaries, hemorrhage can accumulate within the bullae. Recognizing prominent upper dermal hemorrhage as a secondary change may lead to a prompt diagnosis of LSA. We present a case of extragenital LSA that mimics a dermal hemorrhage clinically and histologically in a 71-year-old Caucasian woman.
\end{abstract}

Categories: Dermatology

Keywords: lichen sclerosus et atrophicus, bullous, hemorrhagic, lsa

\section{Introduction}

Lichen sclerosus et atrophicus (LSA) is a chronic inflammatory condition often characterized by atrophic and ivory-white sclerotic plaques in both genital and extragenital regions. Rare variants, such as bullous and hemorrhagic forms of LSA, are often more difficult to recognize both clinically and histopathologically. Histologically, fully developed lesions of LSA are characterized by a thinned, effaced epidermis with interface change, a wide band of hyalinization in the upper dermis, and a lymphohistiocytic infiltrate below the hyalinized area. Importantly, hemorrhagic or bullous changes may be the predominant feature of LSA. Extensive vacuolar degeneration weakens the integrity of the dermoepidermal junction, which contributes to the development of marked edema in the papillary dermis and subepidermal vesiculation. With increased fragility of dermal capillaries, hemorrhage can accumulate within the bullae [1].

Received 06/01/2020 Review began 06/12/2020 Review ended 07/15/2020 Published 07/22/2020

(c) Copyright 2020 Phan et al. This is an open access article distributed under the terms of the Creative Commons Attribution License CC-BY 4.0., which permits unrestricted use, distribution, and reproduction in any medium, provided the original author and source are credited.

Recognizing prominent upper dermal hemorrhage as a secondary change may lead to a prompt diagnosis of LSA. We present a case of extragenital LSA that mimics a dermal hemorrhage clinically and histologically in a 71-year-old Caucasian woman, highlighting this diagnostic pitfall of LSA.

\section{Case Presentation}

A 71-year-old Caucasian female patient with no prior history of skin cancer presented with a red, painful, and enlarging plaque on her right breast for several weeks. This lesion has not been treated in the past and she denies genital lesions.

Physical exam revealed a hemorrhagic plaque with subtle surrounding atrophic changes on her right medial breast (Figure 1). Punch biopsy of the plaque revealed hyperkeratosis with epidermal atrophy, prominent papillary dermal edema with homogenization, and extensive hemorrhage at the dermoepidermal junction, suggestive of LSA (Figure 2). Unfortunately, the patient was lost to follow-up and did not receive treatment. 


\section{Cureus}

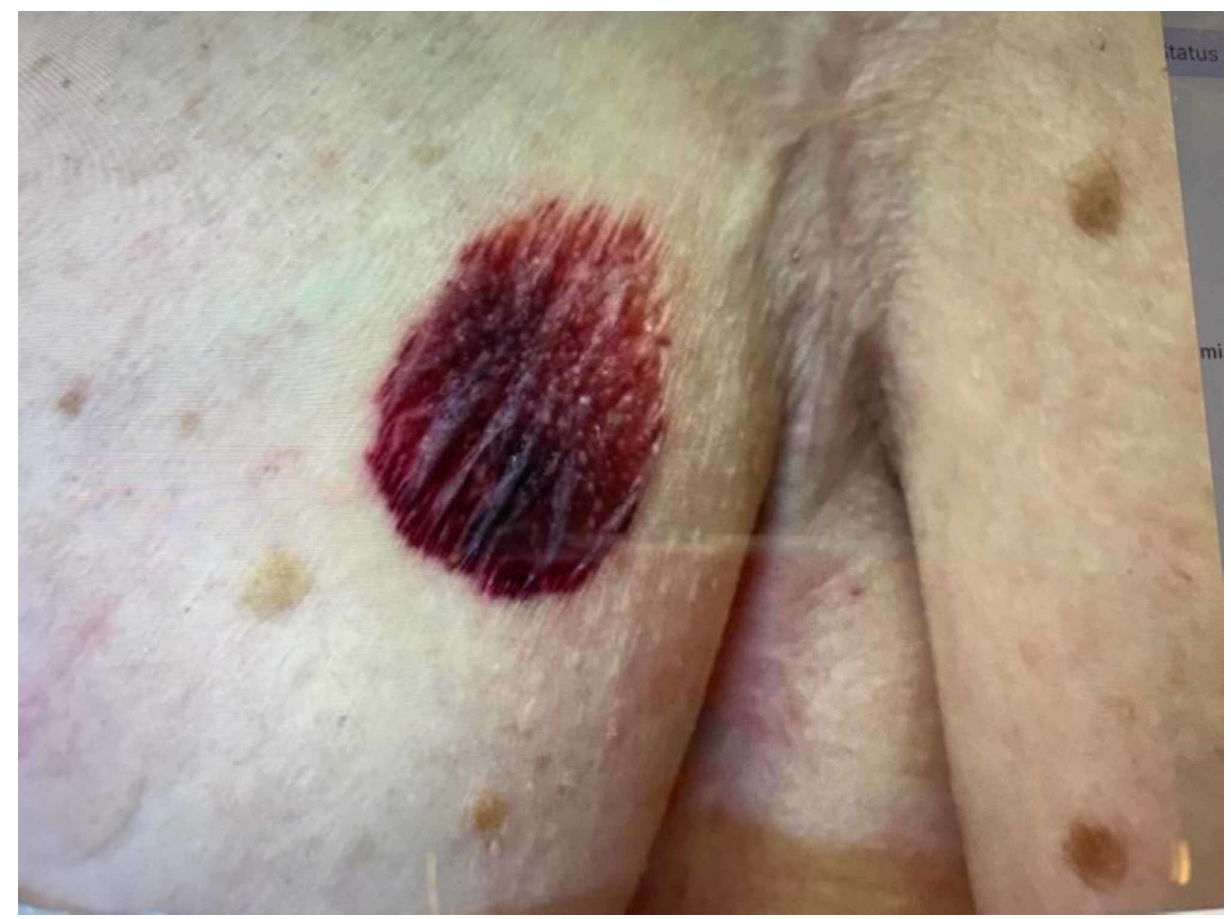

FIGURE 1: Clinical image of right medial breast demonstrating a welldemarcated fragile hemorrhagic plaque with thin cigarette paper-like texture, with surrounding atrophic skin.

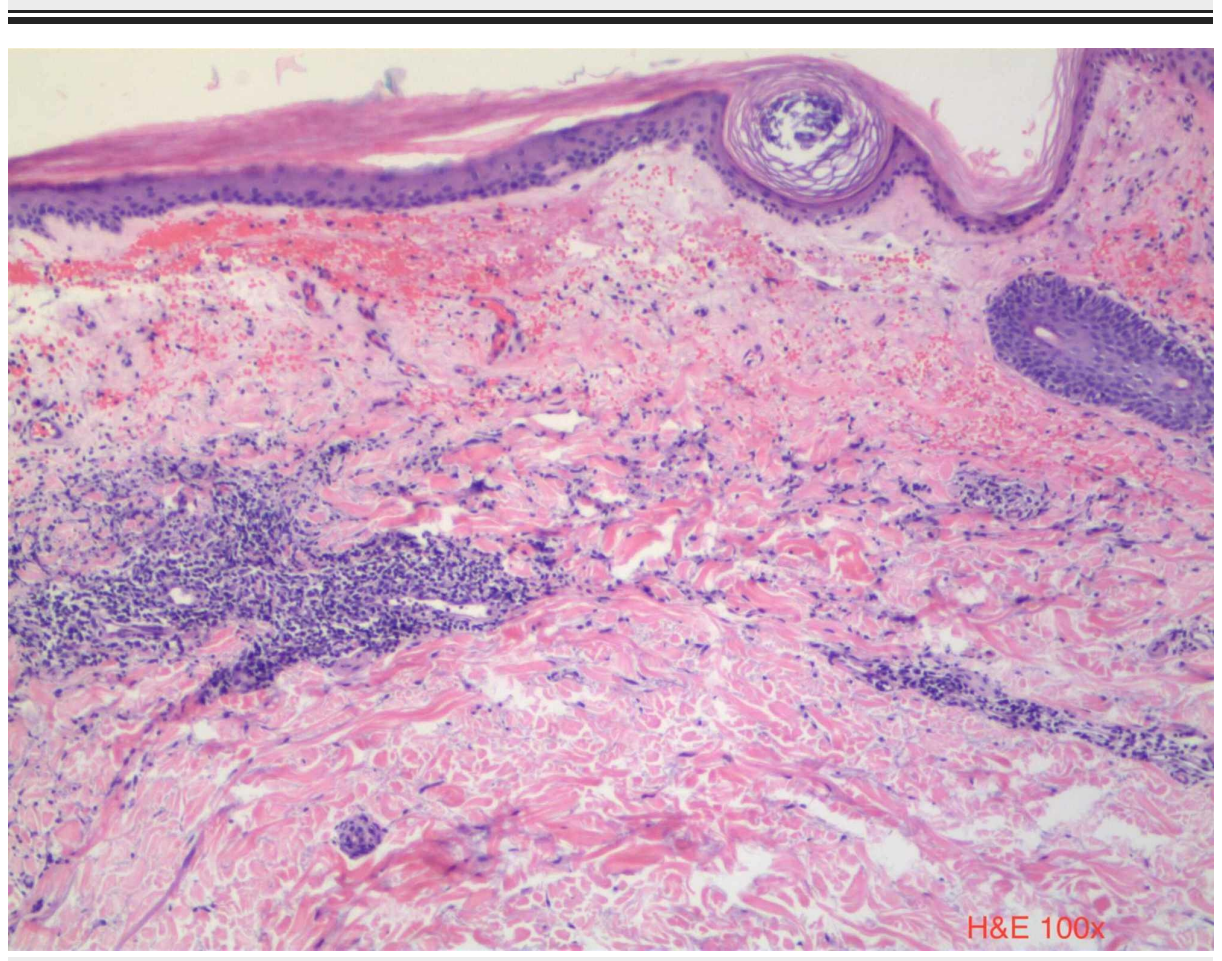

FIGURE 2: H\&E $\times 100$ histology demonstrating hyperkeratosis, epidermal atrophy, papillary dermal edema, and band-like lymphocytic infiltrates directly below. Notice the subepidermal vesiculation and homogenization of the papillary dermis surrounding the hemorrhage.

\section{Discussion}




\section{Pathogenesis}

Bullous/hemorrhagic LSA is a severe and rare variant of LSA. This variant of LSA stems from prominent hydropic degeneration of the basal membrane. This feature is also closely associated with marked papillary dermal edema and even with subepidermal vesiculation and hemorrhage. Although the etiology of bullous LSA is unknown, loss of androgen receptor expression, random inactivation of the androgen receptor gene, autoimmunity with type II diabetes mellitus, and Borrelia infection are considered possible causes [1,2]. The extragenital form of LSA occurs in approximately $20 \%$ of the patients, and the isolated extragenital lichen sclerosus being even rarer tends to develop unusual clinical presentation, such as the development of vesiculobullous lesions [3]. Multiple authors have reported cases of extragenital of LSA trunk, neck, proximal extremities of arms, scalp, and even orofacial involvement [4-7]. Patients with extragenital hemorrhagic and/or bullous LSA can either present with localized isolated plaques or more extensive lesions with the latter being more difficult to treat. Bullous and non-bullous extragenital LSA have also been reported in association with idiopathic morphea as well as radiation-induced morphea [8,9]. However, overlap between morphea and LSA is unlikely in our patient due to different histopathological features. Morphea is characterized by sclerosis of the reticular dermis, swollen collagen fibers, a perivascular infiltrate, and a loss of adnexal structures. LSA is characterized by follicular plugs and a lichenoid infiltrate in the papillary dermis. Additionally, elastic fibers are normal in morphea but absent in LSA [10].

\section{Diagnosis}

Dermascopy of extragenital LSA may reveal scales, keratotic plugs, chrysalis structures, and erosions [11]. Other unusual accompanied features that have been previously described with LSA include telangiectasia, purpura, angiokeratoma-like lesions, and lymphangiectasis [4]. Differential diagnosis of bullous LSA includes bullous lupus erythematosus, bullous cicatricial pemphigoid, bullous scleroderma, bullous lichen planus, trauma, and senile purpura [3]. Recognizing that LSA can, in rare occasions, exhibit prominent upper dermal hemorrhage is essential in making the proper diagnosis. Isolated hemorrhagic plaques and/or bullous lesions may appear prior to the appearance of the more typical plaques of LSA, which can confound clinical diagnosis. Histopathological analysis helps differentiate LSA from other clinically mimicking diseases. Because LSA affected lesions have a long-term risk of squamous cell carcinoma development, close patient follow-up is warranted [12].

\section{Management}

There is no definitive treatment for bullous LSA, and no randomized controlled trials have evaluated treatment of extragenital LSA [1,12]. Numerous therapies have been used for LSA, including topical and systemic corticosteroids, topical estrogen and testosterone containing ointments, retinoids, tacrolimus, and psoralen plus ultraviolet. However, many cases of bullous LSA may be refractory to first-line treatment consisting of topical corticosteroids. Kreuter et al. reported that patients with refractory generalized extragenital lichen sclerosus benefited from pulsed high-dose corticosteroids combined with low-dose methotrexate treatment [13]. Di Silverio and Serri reported marked improvement in a patient with generalized bullous and hemorrhagic LSA that was treated with adrenocorticotropic hormone [14]. Patients with vulvar LSA refractory to ultrapotent topical corticosteroid have been reported to achieve remission after undergoing fractional carbon dioxide laser with resurfacing or ablative settings [15]. Further studies are needed to determine an effective treatment option for the bullous variant of LSA.

The clinical presentation during resolution of LSA varies from case to case. Bullous lesions of LSA may involute leaving a hyperkeratotic plaque that often tend to persist and are refractory to usual therapies, such as topical corticosteroid therapy and doxycycline [1]. Lima et al. reported a case of hemorrhagic bullous lesion remission with hyperkeratotic lesions after two months of treatment with high-potency topical corticosteroids [3]. Khatu and Vasani reported a patient whose bullous lesions healed with postinflammatory hypopigmentation and scarring following a short course of oral corticosteroid and topical clobetasol dipropionate $0.05 \%$ cream with tacrolimus ointment $(0.1 \%)$ [16].

\section{Conclusions}

Extensive bullous/hemorrhagic LSA is a severe and rare variant of LSA. This case of extragenital LSA mimicking dermal hemorrhage both clinically and histologically highlights a diagnostic pitfall. Early recognition of such unique extensively hemorrhagic or bullous presentation of LSA can prevent misdiagnosis and inadequate treatment. Further studies are needed to evaluate treatment options for extragenital LSA.

\section{Additional Information \\ Disclosures}

Human subjects: Consent was obtained by all participants in this study. Conflicts of interest: In compliance with the ICMJE uniform disclosure form, all authors declare the following: Payment/services info: All authors have declared that no financial support was received from any organization for the submitted work. Financial relationships: All authors have declared that they have no financial 
relationships at present or within the previous three years with any organizations that might have an interest in the submitted work. Other relationships: All authors have declared that there are no other relationships or activities that could appear to have influenced the submitted work.

\section{References}

1. Vukicevic J: Extensive bullous lichen sclerosus et atrophicus. An Bras Dermatol. 2016, 91:81-83. 10.1590/abd1806-4841.20164398

2. Ristic B, Divic J, Belic LJ, Zdelar D: Bullous lichen sclerosus et atrophicus . J Eur Acad Dermatol Venereol. 2004, 18:108-109. 10.1111/j.1468-3083.2004.00725.x

3. Lima RS, Maquine GA, Schettini AP, Santos M: Bullous and hemorrhagic lichen sclerosus: case report. An Bras Dermatol. 2015, 90:118-120. 10.1590/abd1806-4841.20153502

4. Fernandez-Flores A, Gatica-Torres M, Tinoco-Fragoso F, Garcia-Hidalgo L, Monroy E, Saeb-Lima M: Three cases of bullous morphea: histopathologic findings with implications regarding pathogenesis. J Cutan Pathol. 2015, 42:144-149. 10.1111/cup.12418

5. Kaur S, Thami GP, Kanwar AJ, Mohan H: Linear oro-facial lichen sclerosus. Clin Exp Dermatol. 2002, 27:467470. 10.1046/j.1365-2230.2002.01068.x

6. Walsh SN, Jorizzo JL, Haverstock C, Sangueza OP: A linear orofacial macule. Am J Dermatopathol. 2008, 30:194-195. 10.1097/DAD.0b013e318162eaca

7. Gomez-Calcerrada MR, del Cerro Heredero M, Sanchez MH, Fernandez RS, de Eusebio Murillo E, Yus ES: Bullous and hemorrhagic lesions. Arch Dermatol. 1999, 135:81-86.

8. Tosti A, Melino M, Bardazzi F: Hemorrhagic bullous lesions in morphea . Cutis. 1989, 44:118-119.

9. Petersen E, Yazdani L, Hymes SR: A case of radiation-induced bullous morphea/lichen sclerosus overlap in a breast cancer patient. Rep Pract Oncol Radiother. 2018, 23:47-49.

10. Kim DH, Lee KR, Kim TY, Yoon MS: Coexistence of lichen sclerosus with morphoea showing bilateral symmetry. Clin Exp Dermatol. 2009, 34:416-418. 10.1111/j.1365-2230.2009.03396.x

11. Larre Borges A, Tiodorovic-Zivkovic D, Lallas A, et al.: Clinical, dermoscopic and histopathologic features of genital and extragenital lichen sclerosus. J Eur Acad Dermatol Venereol. 2013, 27:1433-1439. 10.1111/j.1468-3083.2012.04595.x

12. Hasegawa M, Ishikawa O, Asano Y, et al.: Diagnostic criteria, severity classifications, and clinical guidelines of lichen sclerosus et atrophicus. J Dermatol. 2018, 45:891-897. 10.1111/1346-8138.14171

13. Kreuter A, Tigges C, Gaifullina R, Kirschke J, Altmeyer P, Gambichler T: Pulsed high-dose corticosteroids combined with low-dose methotrexate treatment in patients with refractory generalized extragenital lichen sclerosus. Arch Dermatol. 2009, 145:1303-1308. 10.1001/archdermatol.2009.235

14. Di Silverio A, Serri F: Generalized bullous and haemorrhagic lichen sclerosus et atrophicus. Marked improvement with ACTH. Br J Dermatol. 1975, 93:215-217. 10.1111/j.1365-2133.1975.tb06744.X

15. Lee A, Lim A, Fischer G: Fractional carbon dioxide laser in recalcitrant vulval lichen sclerosus . Australas J Dermatol. 2016, 57:39-43. 10.1111/ajd.12305

16. Khatu S, Vasani R: Isolated, localized extragenital bulbous lichen sclerosus et atrophicus: a rare entity . Indian J Dermatol. 2013, 58:409. 10.4103/0019-5154.117351 